\title{
Causes and prognosis of symptomatic pericardial effusions treated by pericardiocentesis in an Asian academic medical centre
}

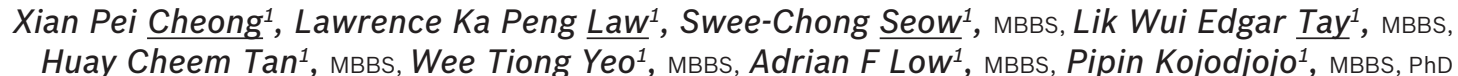

\begin{abstract}
INTRODUCTION This study aimed to investigate the causes, clinical management and outcomes of clinically significant pericardial effusions, and evaluate the practice of pericardiocentesis within an academic medical centre in Singapore, a multiethnic country in Southeast Asia.

METHODS Consecutive patients undergoing pericardiocentesis at a single Asian academic medical centre were identified. Patient demographics, echocardiographic findings, investigations, pericardiocentesis procedural details and clinical progress were tracked using a comprehensive electronic medical records system.

RESULTS Of 149 patients who underwent pericardiocentesis, malignancy (46.3\%) was the most common cause of pericardial effusions, followed by iatrogenic postsurgical complications (17.4\%). $77.3 \%$ of effusions were large and $69.8 \%$ demonstrated tamponade physiology. Pericardiocentesis guided by echocardiography and fluoroscopy was successful in $99.3 \%$ of patients and had a complication rate of $2.0 \%$. Likelihood of effusion recurrence and survival to discharge was determined by the aetiology of the pericardial effusion. $24.6 \%$ of malignant effusions recurred, and the survival rate 12 months after drainage of a malignant pericardial effusion was $45.0 \%$. Short-term mortality was highest among patients presenting with tamponade due to acute aortic syndromes and those with myocardial rupture due to ischaemic heart disease.

CONCLUSION Cancer and iatrogenic complications were the most common causes of pericardial effusion in this large cohort of Singapore patients. Pericardiocentesis has a high success rate and relatively low complication rate. Prognosis and clinical course after pericardiocentesis are determined by the underlying cause of the pericardial effusion.
\end{abstract}

Keywords: pericardial effusion, pericardiocentesis, tamponade

\section{INTRODUCTION}

Pericardial effusions are caused by a wide range of conditions, and the distribution of these causes can vary globally. When complicated by tamponade, pericardiocentesis is potentially a life-saving intervention. However, there is limited data on patients with clinically significant pericardial effusions in Asia. The objectives of this study were to: (a) investigate the causes, clinical management and outcomes of clinically significant pericardial effusions; and (b) evaluate the practice of pericardiocentesis within an academic medical centre in Singapore, a multiethnic country in Southeast Asia.

\section{METHODS}

Consecutive patients undergoing pericardiocentesis at National University Heart Centre, Singapore, (NUHCS) between August 2011 and February 2017 were identified from a comprehensive cardiac procedural database. Patient demographics, echocardiographic findings, investigations, pericardiocentesis procedural details and clinical progress were tracked using a comprehensive electronic medical records system. National University Hospital is a 1,250bed academic medical centre in Singapore that provides tertiary services in oncology (National University Cancer Institute, Singapore) and cardiovascular medicine (NUHCS).
Echocardiographic findings of note that were recorded included ejection fraction, size of the pericardial effusion and the presence of tamponade physiology. The size of the effusion was assessed semi-quantitatively as mild $(<10 \mathrm{~mm})$, moderate (10-20 mm) or large (> $20 \mathrm{~mm}$ ) according to the measure of the largest echo-free space when viewed from standard transthoracic echocardiographic windows. ${ }^{(1)}$ The presence of tamponade was assessed holistically using a range of indicators that included diastolic right heart collapse, abnormal ventricular septal motion, exaggerated inspiratory variability in mitral inflow velocity and inferior vena cava plethora.

Pericardiocentesis was guided either by using echocardiography alone or combined with fluoroscopy in the cardiac catheterisation laboratory, at the operator's discretion. Echocardiographic guidance was used to identify the largest collection of pericardial effusion that was closest to the skin surface without intervening lung or liver tissue. Images were acquired from both the apical and subcostal regions to identify the safest approach. The pericardiocentesis needle was then introduced according to the inclination of the echo probe to the expected depth where the effusion was seen. Occasionally, the needle tip was visualised by the echocardiography probe to confirm entry into the pericardial space, although this was not consistently demonstrated

${ }_{1}^{1}$ Department of Cardiology, National University Heart Centre, Singapore

Correspondence: Dr Pipin Kojodjojo, Consultant, Department of Cardiology, National University Heart Centre, 1E Kent Ridge Road, NUHS Tower Block, Level 9, Singapore 119228. pipin_kojodjojo@nuhs.edu.sg 

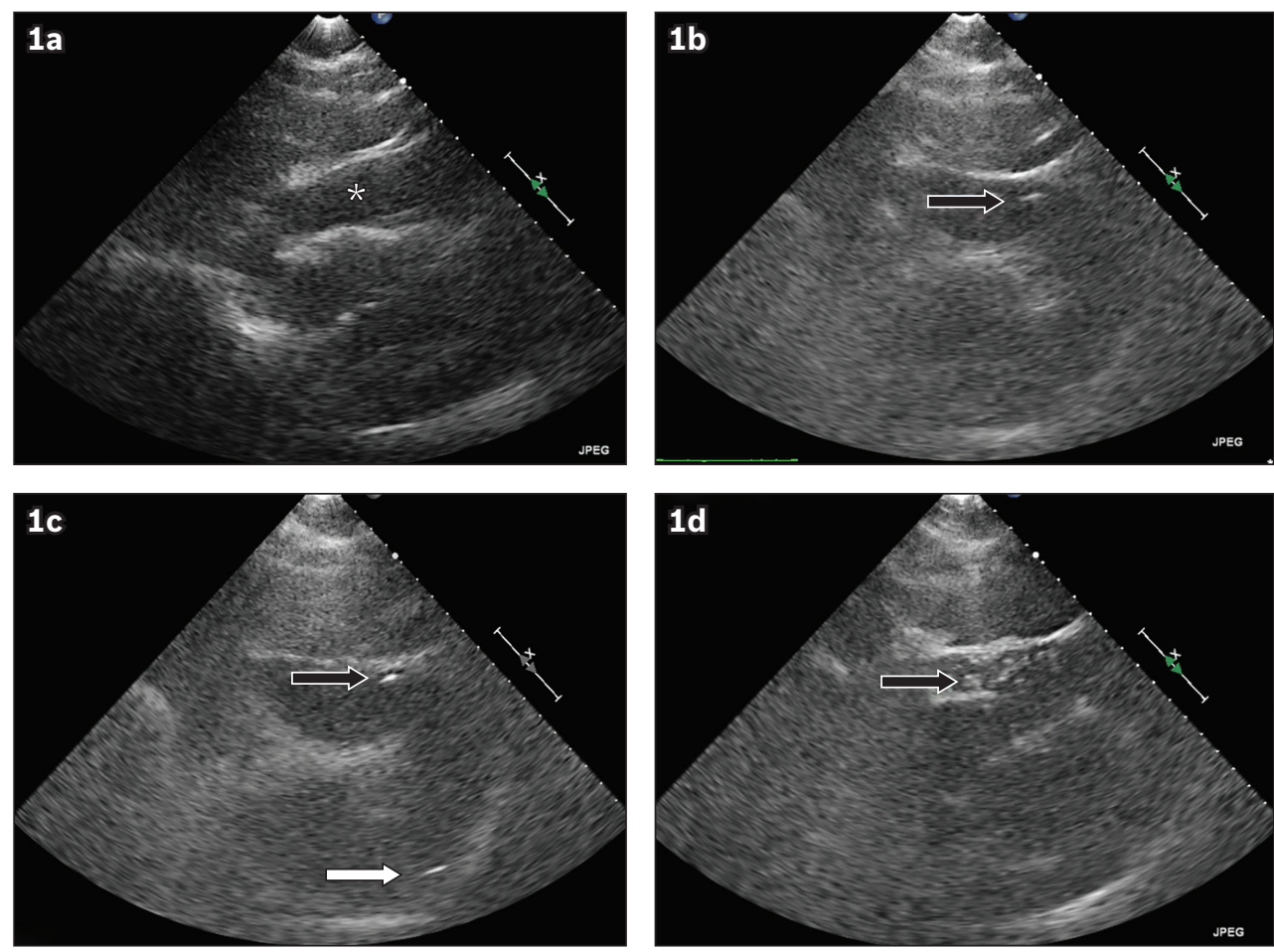

Fig. 1 Echocardiograms of echocardiography-assisted pericardiocentesis show (a) subcostal view of a large pericardial effusion $\left({ }^{*}\right)$ with diastolic collapse of the right heart; (b) visualisation of the needle tip (black arrow) entering the pericardial space; (c) presence of the needle (black arrow) and guidewire (white arrow) within the pericardial space; and (d) presence of echocardiographic bubbles in the pericardial space after injection of agitated saline (black arrow) via a dilator.

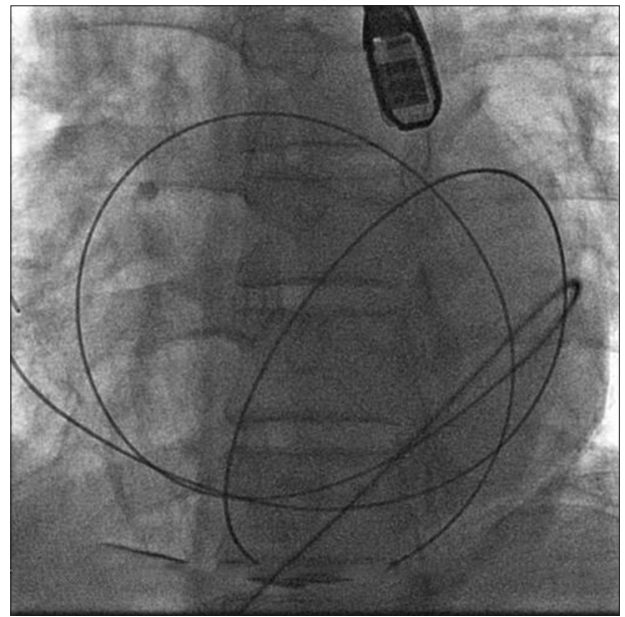

Fig. 2 Fluoroscopic image shows the use of fluoroscopy-guided pericardiocentesis. Note the use of small amounts of contrast to track the progress of the needle from the site of puncture into the pericardial cavity. The position of the guidewire within the pericardial space was confirmed by the passage of the guidewire going around the pericardial sac and traversing multiple anatomical planes, which would not be possible if the guidewire was within the heart.

(Fig. 1). Echocardiographic presence of the guidewire within the pericardial cavity was confirmed prior to the insertion of the dilator. To further ensure correct placement of the sheath, agitated saline (bubble contrast) was injected through the smallest dilator to confirm the presence of bubbles in the pericardial space before further dilatation was performed. For patients undergoing fluoroscopy-guided pericardiocentesis, fluoroscopy in the cardiac catherisation laboratory was used to localise the advancing needle in relation to the cardiac silhouette, combined with contrast injection to determine entry into the pericardial space and to verify the pericardial location of the guidewire by visualising it coiling around the pericardial sac (Fig. 2). It was left to the operator's discretion whether echocardiography was used to complement the fluoroscopic images.

The pericardial fluid underwent cytological, microbiological, microscopic and biochemical analyses, the latter consisting of glucose, protein and lactate dehydrogenase levels. Procedural details during pericardiocentesis were recorded, including site of needle entry (apical vs. subcostal), amount drained, occurrence of any complications and analysis of the pericardial fluid obtained. Causes of pericardial effusion were established based on clinical reasoning and the results of the pericardial fluid analysis. Clinical progress, specifically recurrence of the effusion, need for repeated drainage and survival to discharge, were recorded.

Patients were considered to have active cancer if the disease was diagnosed and treated within the past 12 months and the patient was in remission for less than 12 months. Patients were considered to have previously treated cancer if they were in remission for more than 12 months.

Continuous data was expressed as mean \pm standard deviation. Continuous and categorical variables were assessed with unpaired $t$-test and Fisher's exact test, respectively. GraphPad Prism version 7.0 (GraphPad Software Inc, San Diego, CA, USA) was used for all statistical analyses. The study received institutional ethics approval from the National Healthcare Group Domain Specific Review Board, Singapore (approval no. 2017/00535).

\section{RESULTS}

From August 2011 to February 2017, 149 patients with a mean age of $64.5 \pm 15.7$ years underwent pericardiocentesis (Table I). 
The mean follow-up duration was $10.8 \pm 13.2$ months. $44.3 \%$ of patients were diagnosed with active cancer, and $6.0 \%$ of patients were considered to be in remission for over one year for previously treated cancer. More than half of the cancers originated from the lung. Other common comorbidities included hypertension, hyperlipidaemia and coronary artery disease in $49.7 \%, 43.6 \%$ and $26.2 \%$ of patients, respectively. The most common presenting symptom was dyspnoea, which was reported by $51.0 \%$ of patients.

$81.7 \%$ of patients had left ventricular ejection function within normal limits and $77.3 \%$ of pericardial effusions were large in size (Table II). Tamponade physiology was present in nearly $70 \%$ of patients. In the remaining patients, pericardiocentesis was performed for diagnostic purposes or symptom relief. Loculated effusions were seen in four out of nine patients who developed effusions after cardiac surgery.

Procedural success was achieved in all but one patient, and a mean amount of $520 \pm 408 \mathrm{~mL}$ of fluid was drained during the index procedure (Table III). The subcostal approach was used in $47.7 \%$ of patients. Only $31.5 \%$ of patients had both echocardiography and fluoroscopy guidance. There were $3(2.0 \%)$ cases of serious complications, all of which required surgical intervention - two patients with right ventricular punctures (both using the subcostal approach, one with echocardiographic guidance alone and the other with both echocardiographic and fluoroscopic guidance) and one patient with left ventricular puncture (using an apical approach with echocardiographic guidance). All three patients survived to hospital discharge. In two patients, self-terminating atrial tachyarrhythmias occurred, which did not require any intervention.

$76.9 \%$ of pericardial effusions were bloodstained, while the remainder was described as yellow or serous in appearance. No mycobacterial infection was found in our cohort. Out of 69 patients with malignant pericardial effusions, abnormal cytology was reported for only 31 (44.9\%) patients. On comparing pericardial fluid from malignant effusions (which would be expected to be exudative) against those due to heart failure and uraemia (which would be expected to be transudative), there were no differences in cell counts and protein levels. However, malignant pericardial effusions had lower glucose levels (malignant effusion: $4.0 \pm 2.4 \mathrm{mmol} / \mathrm{L}$; heart failure effusion: $7.0 \pm 5.0 \mathrm{mmol} / \mathrm{L} ; \mathrm{p}<0.01)$ and higher lactate dehydrogenase levels (malignant effusion: 3,181 $\pm 3,879 \mathrm{IU} / \mathrm{L}$; heart failure effusion: $649 \pm 501 \mathrm{IU} / \mathrm{L} ; \mathrm{p}<0.01$ ). There was a significant overlap in both glucose and lactate dehydrogenase levels between the two groups.

The most common cause of pericardial effusion was malignancy, followed by iatrogenic complications of cardiovascular interventions (17.4\%; Table IV). In the latter, five patients were diagnosed following catheter ablation procedures for arrhythmias, four patients after percutaneous coronary arterial interventions, two patients post myocardial biopsy and the remaining 15 patients after cardiac surgery. Recurrences were observed most frequently in patients with malignant effusions $(24.6 \%)$, but repeat drainage was performed in only six out of
Table I. Patient demographics $(n=149)$.

\begin{tabular}{|ll|}
\hline Variable & No. $(\%) /$ mean \pm SD \\
\hline Age (yr) & $64.5 \pm 15.7$ \\
\hline Male gender & $81(54.4)$ \\
\hline Active cancer & $66(44.3)$ \\
\hline Lung & $37(56.1)$ \\
\hline Leukaemia/lymphoma & $12(18.2)$ \\
\hline Breast & $6(9.1)$ \\
\hline Gastrointestinal & $3(4.5)$ \\
\hline Head and neck & $3(4.5)$ \\
\hline Gynaecological & $2(3.0)$ \\
\hline Other & $3(4.5)$ \\
\hline Previously treated cancer & $9(6.0)$ \\
\hline Coronary artery disease & $39(26.2)$ \\
\hline Heart failure & $21(14.1)$ \\
\hline Hypertension & $74(49.7)$ \\
\hline Hyperlipidaemia & $65(43.6)$ \\
\hline Myocarditis & $8(5.4)$ \\
\hline Diabetes mellitus & $23(15.4)$ \\
\hline End-stage renal failure & $19(12.8)$ \\
\hline Chronic lung disease & $10(6.7)$ \\
\hline Liver cirrhosis & $7(4.7)$ \\
\hline Prior tuberculosis & $8(5.4)$ \\
\hline Presenting symptom & $13(20.7)$ \\
\hline Breathlessness/dyspnoea & \\
\hline Chest pain & $30.1)$ \\
\hline Hypotension & \\
\hline Palpitation & $31.0)$ \\
\hline
\end{tabular}

SD: standard deviation

Table II. Echocardiographic characteristics.

\begin{tabular}{|lc|}
\hline Variable & $\%$ \\
\hline Left ventricular ejection fraction & \\
\hline Normal $(\geq 55 \%)$ & 81.7 \\
\hline Impaired $(<55 \%)$ & 18.3 \\
\hline Size of pericardial effusion & 7.1 \\
\hline Small & 15.6 \\
\hline Moderate & 77.3 \\
\hline Large & 30.2 \\
\hline Tamponade physiology & 69.8 \\
\hline No
\end{tabular}

these 17 patients. One patient underwent surgery to create a pericardial window. Survival to discharge was closely related to aetiology, with the lowest survival rates seen among patients with acute ascending aortic syndrome (aortic dissections or rupture of ascending aortic aneurysms) and ventricular free wall rupture following acute myocardial infarctions. In contrast, all patients diagnosed with pericarditis, uraemia, heart failure and autoimmune causes survived to discharge. The survival rate at 12 months after pericardiocentesis for malignant effusions was $45.0 \%$. 
Table III. Procedural details.

\begin{tabular}{|c|c|c|c|}
\hline \multirow[t]{2}{*}{ Variable } & \multicolumn{3}{|c|}{ No. (\%)/mean \pm standard deviation } \\
\hline & All $(n=149)$ & $\begin{array}{l}\text { Echocardiography } \\
\text { guided }(n=102)\end{array}$ & $\begin{array}{l}\text { Echocardiography and } \\
\text { fluoroscopy guided }(n=47)\end{array}$ \\
\hline Acute procedural success & $148(99.3)$ & $101(99.0)$ & $47(100.0)$ \\
\hline Amount drained* $(\mathrm{mL})$ & $520 \pm 408$ & $517 \pm 341$ & $507 \pm 506$ \\
\hline \multicolumn{4}{|l|}{ Site of entry } \\
\hline Apical & $78(52.3)$ & $58(56.9)$ & $20(42.6)$ \\
\hline Subcostal & $71(47.7)$ & $44(43.1)$ & $27(57.4)$ \\
\hline Complication requiring intervention & $3(2.0)$ & $2(2.0)^{*}$ & $1(2.1)^{+}$ \\
\hline
\end{tabular}

${ }^{*}$ One patient had right ventricular puncture and one patient had left ventricular puncture. †One patient had right ventricular puncture.

Table IV. Causes of pericardial effusion, likelihood of recurrence and survival to hospital discharge rate.

\begin{tabular}{|c|c|c|c|}
\hline \multirow[t]{2}{*}{ Variable } & \multicolumn{3}{|c|}{ No. (\%) } \\
\hline & Incidence & Recurrence & Survival to discharge \\
\hline Malignancy & $69(46.3)$ & $17(24.6)$ & $50(72.5)$ \\
\hline Postsurgical complications & $26(17.4)$ & $1(3.8)$ & $20(76.9)$ \\
\hline Effusion associated with pneumonia & $11(7.4)$ & $2(18.2)$ & $8(72.7)$ \\
\hline Pericarditis & $9(6.0)$ & $2(22.2)$ & $9(100.0)$ \\
\hline Uraemia & $7(4.7)$ & $1(14.3)$ & $7(100.0)$ \\
\hline Acute ascending aortic syndrome & $7(4.7)$ & $0(0)$ & $3(42.9)$ \\
\hline Heart failure & $6(4.0)$ & $0(0)$ & $6(100.0)$ \\
\hline Multisystemic autoimmune disease & $5(3.4)$ & $1(20.0)$ & $5(100.0)$ \\
\hline Idiopathic pericardial effusion & $3(2.0)$ & $0(0)$ & $3(100.0)$ \\
\hline Dressler's syndrome & $3(2.0)$ & $0(0)$ & $3(100.0)$ \\
\hline Over-anticoagulation & $1(0.7)$ & $0(0)$ & $0(0)$ \\
\hline Overall & $149(100.0)$ & $24(16.1)$ & $114(76.5)$ \\
\hline
\end{tabular}

\section{DISCUSSION}

In our series of 149 patients undergoing pericardiocentesis, malignancy followed by iatrogenic postsurgical complications were the most common causes of pericardial effusions. Pericardiocentesis guided by echocardiography and fluoroscopy has a high success rate and relatively low complication rate. However, serious complications requiring surgical interventions may occur. Investigations routinely ordered for analysis of pericardial fluids have limited sensitivity. Our study found that effusion recurrence and survival after pericardiocentesis is determined by the aetiology of the pericardial effusion.

Causes of pericardial effusion vary depending on the geographical location of the survey. In less developed countries such as South Africa, infectious causes such as tuberculosis predominate. ${ }^{(2)}$ Conversely, in advanced countries, complications of cardiac surgery and cancer are the most common causes. ${ }^{(3-5)}$ Furthermore, the proportions of pericardial effusions caused by malignancy and postsurgical complications may be increasing over time, in part due to improved survival among cancer patients and the increasing number of cardiac interventions in developed countries. ${ }^{(6)}$

The prognosis of pericardial effusion is essentially related to its aetiology ${ }^{(1,7)}$ Consistent with other series, patients with malignant pericardial effusions have a poor prognosis with typical median survival of $3-7$ months. ${ }^{(3-5,7)}$ This is consistent with the $45.0 \%$ survival rate at 12 months in our patients. However, patients presenting with haemopericardium due to acute ascending aortic syndrome, which consists of aortic dissections or rupture of aortic aneurysms, and free wall myocardial rupture had the worst shortterm prognosis despite undergoing emergent surgery.

Our major complication rate of $2.0 \%$ was consistent with other large series, confirming the relative safety of pericardiocentesis. ${ }^{(4,8)}$ Given the low incidence of complications, it was not possible to discern whether the site of pericardial access or the additional use of fluoroscopy for guidance impacted complication rates. However, while complications are uncommon, three patients with incidental ventricular puncture required surgical repair in our series. This observation should be a cautionary note about performing non-emergent pericardiocentesis in hospitals without cardiothoracic surgical support.

While pericardial fluid is routinely sent for cell count, measurements of glucose, protein and lactate dehydrogenase levels, and cytology and microbiological analyses, diagnostic yields are limited. All tests listed above, except cytology, generally lack specificity, with significant overlap between transudative and exudative effusions. ${ }^{(9-11)}$ Conversely, cytological analyses have lower sensitivity (only $44.9 \%$ in this study) but $100 \%$ specificity. The positive diagnostic yield of $44.9 \%$ from cytological analysis 
of malignant pericardial effusion in our study was consistent with the rate of $40 \%-50 \%$ in the literature. ${ }^{(5)}$ Interpretations of analyses of pericardial fluid are also limited by: first, the fact that most causes of pericardial effusion would be expected to produce an exudative, typically bloodstained effusion $(76.9 \%$ in this series) and secondly, that the biochemical composition of physiological pericardial fluid differs from that of plasma serum. ${ }^{(12)}$ In a recent study, physiological pericardial fluid was collected from 30 patients undergoing elective coronary artery bypass grafting without a history of pericardial disease. Surprisingly, pericardial lactate dehydrogenase levels were on average 2.4 times higher than serum levels, while protein levels were only $60 \%$ of serum levels. ${ }^{(12)}$ There was also predominant lymphocytosis, which was 5.3 times higher than paired serum levels. This has led to several groups suggesting that analysis of pericardial biochemistry may be unhelpful or have almost no diagnostic value in the contemporary management of pericardial effusions. ${ }^{(10,13)}$

Our study was limited by its retrospective design, lack of a systematic protocol for pericardiocentesis and advanced investigations of the pericardial fluid. However, the findings reflect real-world practices and are informative for future improvements in clinical care. Our results may have been biased due to the presence of tertiary cancer and cardiovascular surgical services within our institution, which resulted in higher proportions of patients with malignant and postsurgical effusions being referred for pericardiocentesis.

In conclusion, cancer and iatrogenic complications are the most common causes of pericardial effusion in this large cohort of Singapore patients. Pericardiocentesis has a high success rate and relatively low complication rate. Prognosis and clinical course after pericardiocentesis are determined by the underlying cause of the pericardial effusion.

\section{REFERENCES}

1. Adler Y, Charron P, Imazio M, et al; ESC Scientific Document Group. 2015 ESC Guidelines for the diagnosis and management of pericardial diseases: the Task Force for the Diagnosis and Management of Pericardial Diseases of the European Society of Cardiology (ESC). Endorsed by: the European Association for Cardio-Thoracic Surgery (EACTS). Eur Heart J 2015; 36:2921-64.

2. Reuter H, Burgess LJ, Doubell AF. Epidemiology of pericardial effusions at a large academic hospital in South Africa. Epidemiol Infect 2005; 133:393-9.

3. Lindenberger M, Kjellberg M, Karlsson E, Wranne B. Pericardiocentesis guided by 2-D echocardiography: the method of choice for treatment of pericardial effusion. J Intern Med 2003; 253:411-7.

4. Akyuz S, Zengin A, Arugaslan E, et al. Echo-guided pericardiocentesis in patients with clinically significant pericardial effusion. Outcomes over a 10-year period. Herz 2015; 40 Suppl 2:153-9.

5. Sánchez-Enrique C, Nuñez-Gil IJ, Viana-Tejedor A, et al. Cause and long-term outcome of cardiac tamponade. Am J Cardiol 2016; 117:664-9.

6. Ma W, Liu J, Zeng Y, et al. Causes of moderate to large pericardial effusion requiring pericardiocentesis in $140 \mathrm{Han}$ Chinese patients. Herz 2012; 37:183-7.

7. Kil UH, Jung HO, Koh YS, et al. Prognosis of large, symptomatic pericardial effusion treated by echo-guided percutaneous pericardiocentesis. Clin Cardiol 2008; 31:531-7.

8. Tsang TS, Barnes ME, Hayes SN, et al. Clinical and echocardiographic characteristics of significant pericardial effusions following cardiothoracic surgery and outcomes of echo-guided pericardiocentesis for management: Mayo Clinic experience, 1979-1998. Chest 1999; 116:322-31.

9. Meyers DG, Meyers RE, Prendergast TW. The usefulness of diagnostic tests on pericardial fluid. Chest 1997; 111:1213-21.

10. Ben-Horin S, Bank I, Shinfeld A, et al. Diagnostic value of the biochemical composition of pericardial effusions in patients undergoing pericardiocentesis. Am J Cardiol 2007; 99:1294-7.

11. Karatolios K, Pankuweit S, Maisch B. Diagnostic value of biochemical biomarkers in malignant and non-malignant pericardial effusion. Heart Fail Rev 2013; 18:337-44

12. Ben-Horin S, Shinfeld A, Kachel E, Chetrit A, Livneh A. The composition of normal pericardial fluid and its implications for diagnosing pericardial effusions. Am J Med 2005; 118:636-40.

13. Akyuz S, Arugaslan E, Zengin A, et al. Differentiation between transudate and exudate in pericardial effusion has almost no diagnostic value in contemporary medicine. Clin Lab 2015; 61:957-63. 